\title{
Multi-phosphorylation Of The Intrinsically Disordered Unique Domain Of c- Src Studied By In-cell and Real-Time NMR
}

\author{
Irene Amata ${ }^{[\mathrm{a}],[\mathrm{b}]}$, Mariano Maffei ${ }^{[\mathrm{al}]}$, Ana Igea ${ }^{[\mathrm{b}]}$, Marina Gay ${ }^{[\mathrm{c}]}$, Marta Vilaseca ${ }^{[\mathrm{c}]}$, \\ Angel R. Nebreda ${ }^{[\mathrm{b}],[\mathrm{d}]^{*}}$, Miquel Pons ${ }^{[\mathrm{a}]^{*}}$
}

Intrinsically disordered regions (IDRs) are preferred sites for posttranslational modifications essential for re-programming protein function. The enhanced local mobility of IDRs facilitates their observation by NMR in vivo. Phosphorylation events may occur in multiple sites and respond dynamically to changes in kinasephosphatase networks. Here we used real-time NMR to study the effect of kinases and phosphatases present in Xenopus oocytes and egg extracts on the phosphorylation state of the Unique domain of c-Src. We followed the phosphorylation of $S 17$ in oocytes, and S17, S69 and S75 in egg extracts by NMR, mass spectrometry and western blotting. Addition of specific kinase

Introduction

Post-translational modifications (PTMs) are crucial for cells to be able to quickly reprogram protein functions. Reversible phosphorylation, in particular, is a major regulatory event in signaling pathways. Phosphorylation of serine and threonine residues is the most abundant and the phosphorylation state of particular sites is the result of a delicate balance between kinases and phosphatases, many of which are also regulated by phosphorylation. The presence of multiple phosphorylation sites in the same protein is quite common $^{[1]}$. In these cases, the phosphorylation of one site may prevent or enhance the phosphorylation of others and the order of phosphorylation may determine the final pattern. Thus, the phosphorylation of proteins at multiple sites is best studied in real time and in intact cellular systems ${ }^{[1]}$. Moreover, the use of proteins without PTMs produced in bacteria provides a way to follow how the endogenous kinasephosphatase machinery establishes relevant phosphorylation patterns. Serine and threonine phosphorylation causes very large shifts in Nuclear Magnetic Resonance (NMR) chemical shifts of the corresponding amide protons ${ }^{[2]}$ due to intra-residue hydrogen bonding between amide protons and phosphate moieties ${ }^{[3]}$. The non-invasive character of NMR and the selectivity provided by isotopic enrichment allow the recording of spectra of isotopeenriched proteins in living cells ${ }^{[4],[5],[6]}$. Although steric crowding is

inhibitors showed that S75 and S69 were phosphorylated by CDKs (Cyclin-dependent kinases) different from Cdk1. Moreover, although PKA (cAMP-dependent protein kinase) can phosphorylate S17 in vitro, this was not the major S17 kinase in egg extracts. Changes in PKA activity affected the phosphorylation levels of $C D K$-dependent sites, suggesting indirect effects through kinasephosphatase networks. This study provides a proof-of-concept for the use of real-time in vivo NMR to characterize kinase/phosphatase effects on intrinsically disordered regulatory domains.

expected to cause only minor increases in the correlation time of proteins inside the cytoplasm ${ }^{[7]}$, non-specific interactions with other cell components often result in restricted rotation and broad lines for globular proteins ${ }^{[8]}$. In contrast, local motion in intrinsically disordered proteins or protein fragments usually results in narrow in cell NMR line widths.

Intrinsically disordered regions (IDRs) are highly abundant in proteins of eukaryotic organisms, but not in prokaryotes, suggesting that their appearance is linked to the increased regulatory demands of multicellular organisms $s^{[9],[10]}$ and exploited the reduced tendency of intrinsically disordered proteins (IDPs) to form amyloids ${ }^{[11]}$.

[a] Dr. I. Amata, M. Maffei, Pr. Dr. M. Pons

Biomolecular NMR Laboratory. Department of Organic Chemistry

University of Barcelon

Baldiri Reixac, 10-12

08028- Barcelona. Spain

[b] Dr. I. Amata, Dr. A. Igea, Dr. A.R. Nebreda*

Signaling and Cell Cycle Laboratory, Institute for Research in Biomedicine (IRB Barcelona). Barcelona, Spain

[c] Dr. M. Gay, Dr. M. Vilaseca

Mass Spectrometry Core Facility, Institute for Research in Biomedicine (IRB Barcelona). Barcelona, Spain

[d] Dr. A.R. Nebreda

Institució Catalana de Recerca i Estudis Avançats (ICREA), Barcelona, Spain

*Corresponding authors: mpons@ub.edu; angel.nebreda@irbbarcelona.org

Supporting information for this article is available on the WWW under http://www.chembiochem.org or from the author. 
Indeed, IDRs are often associated with cell regulation and signaling, and $80 \%$ of the proteins involved in human cancers contain long $I \mathrm{DRs}^{[12,13]}$. PTMs sites are often located in exposed; flexible segments and IDRs often display phosphorylation $\operatorname{sites}^{[14]}$.

c-Src is the leading member of a family of non-receptor tyrosine kinases implicated in many cellular functions ${ }^{[15],[16],[17],[18],[19]}$. The cSrc protein is formed by a N-terminal myristoylated membraneanchoring region ( $\mathrm{SH} 4$ domain), followed by an IDR called the Unique domain, a SH3, a SH2 and the kinase, or $\mathrm{SH} 1$, domain.

The Unique domain contains several sites susceptible to be phosphorylated and dephosphorylated in response to diverse cellular processes ${ }^{[20],[21],[22]}$. Although the phosphorylation of $\mathrm{S} 17$ by PKA (cAMP-dependent protein kinase) is a well-characterized process, its biological significance remains obscure ${ }^{[23]}$. Other phosphorylation sites within the Unique domain that have been identified and are believed to modulate the activity of the enzyme are T34, T46, and S72 in chicken c-Src, which correspond to T37 and S75 in human c-Src (while T46 has no correspondence in humans). Phosphorylation of these residues by cyclin-dependent kinase 1 (Cdk1/Cdc2) was first observed during mitosis ${ }^{[24]}$. S75 is not present in other SFKs members, while it is evolutionarily conserved in Src. Mitosis-independent phosphorylation of S75 by Cdk5 was observed in neurons and in certain tumor cell lines ${ }^{[25]}$. Phosphorylation of Src at S75 by Cdk5 is a crucial mechanism for the regulation of intracellular Src activity, as it is directly implicated in modulating the ubiquitin-dependent degradation of active $\mathrm{Src}^{[26]}$.

A major regulatory role of the Unique domain of $\mathrm{c}-\mathrm{Src}$ has been recently demonstrated ${ }^{[27]}$. The discovery followed observations of a number of intra-molecular and inter-molecular interactions involving the Unique domain and lipids or proteins. The biological relevance of these interactions was confirmed in Xenopus laevis oocytes. In this model system we could confirm that mutations in the residues of the Unique domain abolishing lipid binding caused a lethal phenotype in the matured oocytes. Moreover, in vitro studies showed that the phosphorylation of S17, T37 and S75 modulate lipid binding by the Unique domain ${ }^{[28]}$. These phosphorylation sites are highly conserved in Src of phylogenetically distant species, and in particular between human and X. laevis.

Here we present an NMR study of the phosphorylation of the c-Src Unique domain injected in Xenopus oocytes or in the presence of extracts from unfertilized Xenopus eggs, which contain a rich repertoire of kinases and phosphatases. This study constitutes a proof-of-concept of the potential of time-resolved NMR in cells or cell-extracts, alone or in combination with mass-spectrometry and biochemical methods, to study multiple phosphorylationdephosphorylation events of intrinsically disordered regulatory domains of signaling proteins. The methods presented allow the direct observation of the integration of multiple-phosphorylation regulatory processes in the complex kinase-phosphatase networks active under particular physiological or pathological environments.

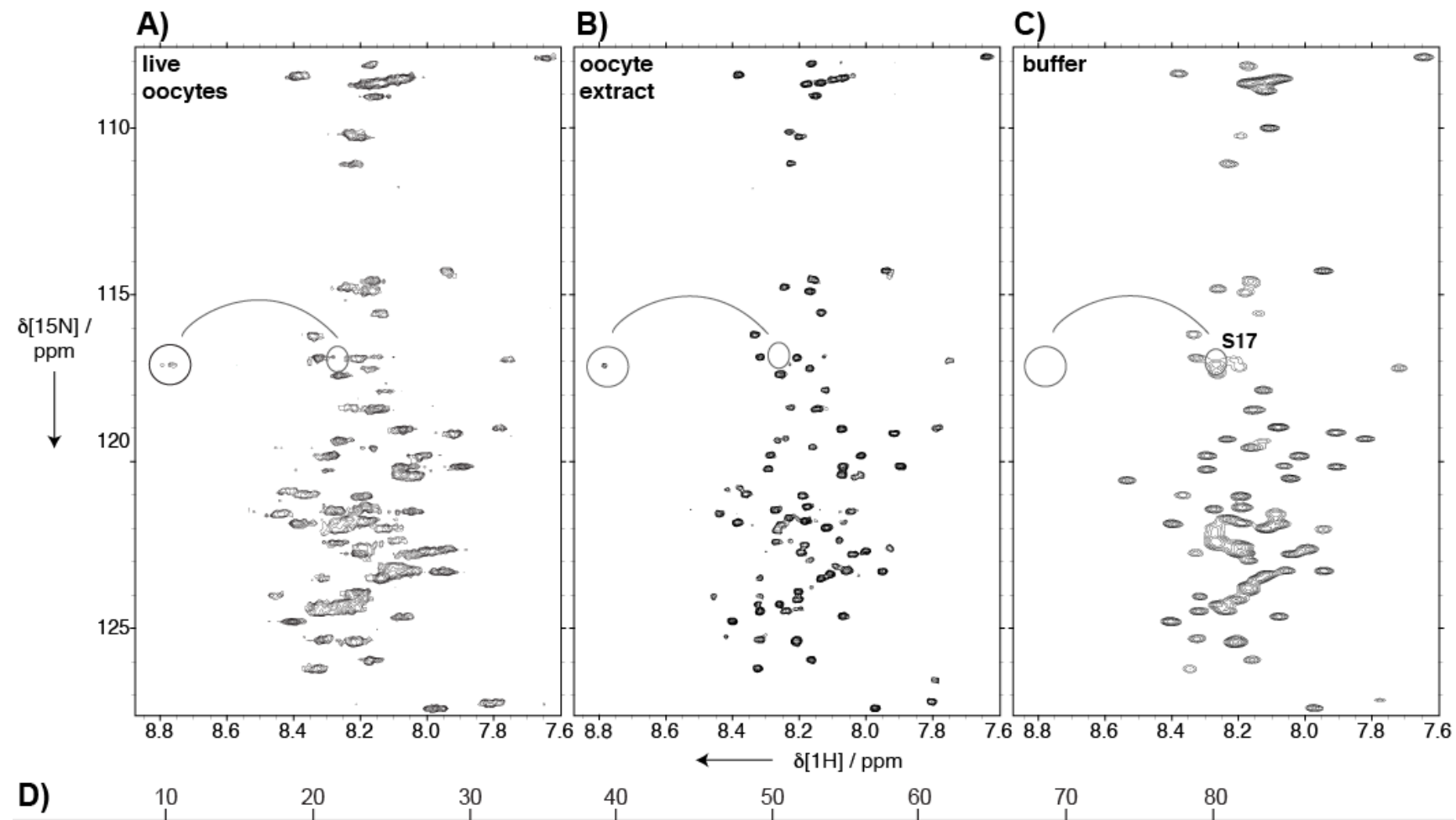

Figure 1. ${ }^{1} \mathrm{H}-{ }^{15} \mathrm{~N}$ correlation NMR spectra of USrc injected in: intact Xenopus oocytes (A), in cytoplasmic extract obtained from the injected oocytes (B), and dissolved in buffer (C). Circles mark the position of pS17. The curved line indicates the shift to the left from unmodified (ovals) to phosphorylated S17. (D) Amino acid sequence of USrc. Strep-tag residues are indicated in Italic. 


\section{Results and Discussion}

The Unique domain of c-Src gives well resolved NMR spectra in Xenopus oocytes: Phosphorylation by endogenous kinases

A ${ }^{15} \mathrm{~N}$-labelled construct, which we refer to as USrc, with the first 85 residues of human c-Src followed by a Strep-tag sequence, was expressed in E.coli and purified as previously described ${ }^{[28]}$. This construct contains the $\mathrm{SH} 4$ and Unique domains. The glycine residue following the initial methionine was mutated to alanine to prevent myristoylation after injection into the oocytes. Figure 1 shows ${ }^{1} \mathrm{H}-{ }^{15} \mathrm{~N}$ correlations of the NMR spectra of USrc either injected in intact Xenopus oocytes (Fig. 1a), in cytoplasmic extract obtained from the injected oocytes (Fig. 1b), or dissolved in buffer (Fig. 1c). Well-resolved NMR spectra were obtained under the three conditions. Most of the resonances of USrc appeared at very similar frequencies, thus making their assignment within the cellular environment straightforward. Comparison of HSQC spectra in oocytes or extracts with those from ${ }^{15} \mathrm{~N}$-USrc re-isolated by Streptag affinity purification after the NMR experiments ruled out degradation within intact oocytes or in extracts of Xenopus oocytes or unfertilized eggs (results not shown).

\section{A)}
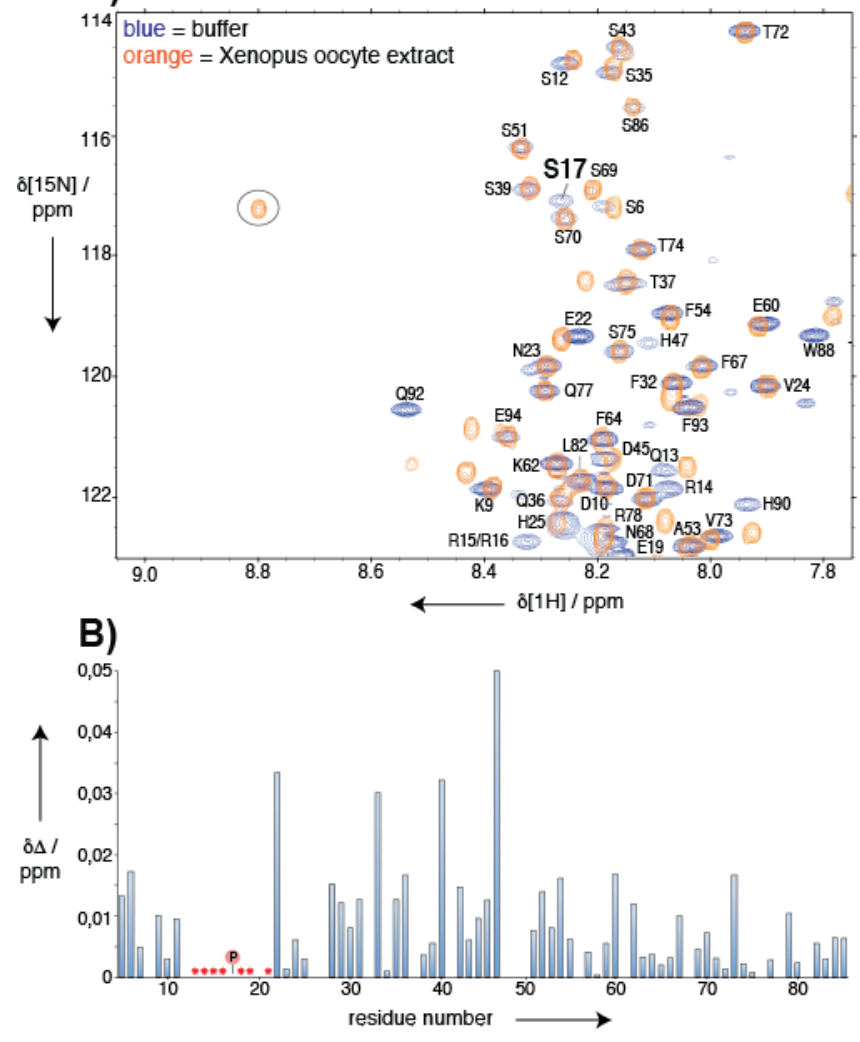

Figure 2. A. Overlay of expansions of ${ }^{1} \mathrm{H}-{ }^{15} \mathrm{~N}$ HSQC spectra of USrc in buffer (blue) and in Xenopus oocytes extract (orange). The circle marks the position of pS17. B. Chemical shift changes between the two conditions. Red asterisks indicate residues with large perturbation preventing their unambiguous assignment in the phosphorylated sample. The phosphorylation site is indicated.
In vitro studies had shown that the Unique domain contains a secondary lipid-binding region, in addition to the $\mathrm{SH} 4$ lipid-anchoring moiety ${ }^{[27]}$.The observation of well-resolved spectra in the intact oocyte suggests that interactions involving this secondary site are weak, at least in the absence of myristoylation.

A major difference was the appearance of a new peak, which, on the basis of its chemical shift, corresponds to a phosphorylated serine or threonine ${ }^{[2]}$. The appearance of this peak matched the disappearance of the signal from $\mathrm{S} 17$, thus indicating that this serine residue is spontaneously phosphorylated in Xenopus oocytes. Similar results were observed when USrc was added to extracts containing the soluble fraction of lysed oocytes, indicating that the phosphorylation involved a kinase that is soluble or at least associated to the soluble lipid fraction (Fig. 2a). S17 can be phosphorylated in vitro by purified $\mathrm{PKA}^{[28]}$, and this kinase is known to be active in Xenopus oocytes ${ }^{[29]}$.

Chemical shift changes between ${ }^{1} \mathrm{H}_{-}{ }^{15} \mathrm{~N}$ HSQC spectra in buffer and
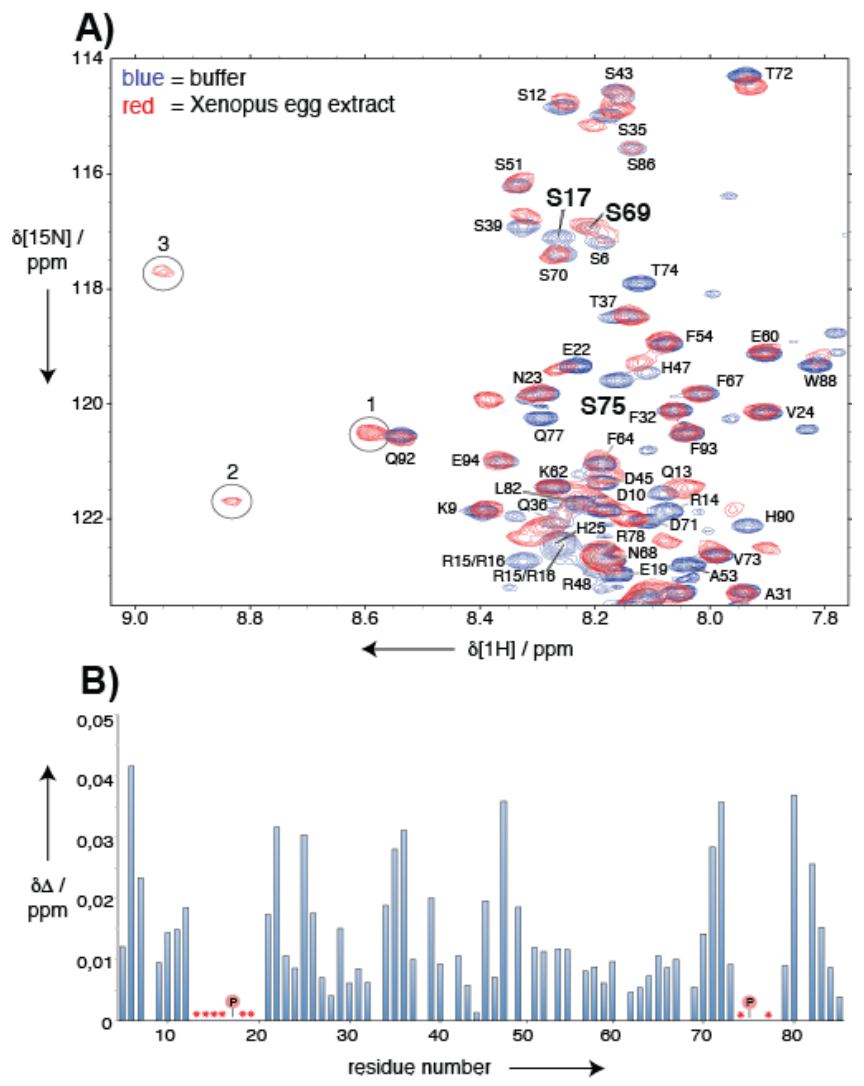

Figure 3. A. Overlay of expansions of ${ }^{1} \mathrm{H}-{ }^{15} \mathrm{~N}$ HSQC spectra of USrc in buffer (blue) and in Xenopus egg extract (CSF extract) (red). The circles mark the signals from phosphorylated serines/threonines. B. Chemical shift changes between the major species present in the two conditions. Red asterisks indicate residues with large perturbation preventing their unambiguous assignment in the phosphorylated sample. The phosphorylation sites unambiguously assigned by NMR are indicated. 
in oocyte extracts are shown in Figure 2b. While most residues displaying small changes were assigned by reference to the spectra obtained in buffer, resonances from residues Q13, R14, R15 or R16, S17, L18, E19 and A21 were significantly shifted or have disappeared and could not be assigned in the oocyte extracts. These residues clustered around phosphorylated S17 and included a number of charged residues probably affected by the presence of the additional charge of the phosphate group. Residue E22, another charged residue close to $\mathrm{S} 17$, also showed chemical shift changes above the standard deviation $(0.13 \mathrm{ppm})$ of the variations observed between buffer and oocyte extracts. Likewise, the charge-sensitive residue $\mathrm{H} 47$ and its neighbor $\mathrm{G} 46$, although located far from $\mathrm{S} 17$ were also significantly perturbed in the oocyte extracts. Interestingly, hydrophobic residues A27 and F32 in the intermediate region were also affected. USrc phosphorylated at S17 showed only very small perturbations around $\mathrm{H} 47$ in buffer ${ }^{[28]}$, thereby suggesting that the perturbations observed in the oocyte extract are mediated by the interaction with some components of the extract. Residues R48, N68 and R78 could not be distinguished in the cell extract due to spectral overlap.

\section{Time-resolved multiple phosphorylations of USrc in Xenopus egg extracts}

Extracts obtained from unfertilized $X$.laevis eggs are referred to as Cytostatic factor (CSF) extracts. Xenopus eggs are arrested in metaphase II of meiosis and present a larger number of active kinases than oocytes, including members of the cyclin dependent kinase (CDK) family ${ }^{[30]}$. We and others showed that S75 in the Unique domain of c-Src can be phosphorylated by active Cdk5 both in vitro and in vivo ${ }^{[26],[28]}$. Figure 3a shows the comparison between ${ }^{1} \mathrm{H}-{ }^{15} \mathrm{~N}$ HSQC correlation spectra of ${ }^{15} \mathrm{~N}$ labeled USrc in buffer and in the Xenopus egg extracts.

After prolonged incubation of ${ }^{15} \mathrm{~N}$-labeled USrc in the X.laevis egg extracts, three signals with chemical shifts typical of phosphorylated Ser/Thr were observed at the following ${ }^{1} \mathrm{H},{ }^{15} \mathrm{~N}$ chemical shift positions (in ppm): 8.596, 120.48 (peak 1), 8.840, 121.76 (peak 2) and 8.96, 117.72 (peak 3) (marked in Fig. 3a). However, the three new peaks appeared at different times with different growing rates The NMR spectra correspond to the major species present after phosphorylation and may not include the effects of minor phosphorylated forms. A number of peaks from non-phosphorylated residues changed chemical shifts. The chemical shift perturbations of USrc residues in the major species present after reaching the steady state ( 3 hours after the reaction started) in the egg extract are shown in Figure $3 \mathrm{~b}$.

In order to increase the time resolution in the observation of the multiple phosphorylation events of USrc, we optimized the experimental conditions by using a combination of SOFAST-HMQC to minimize the recycle time between successive NMR scans, and
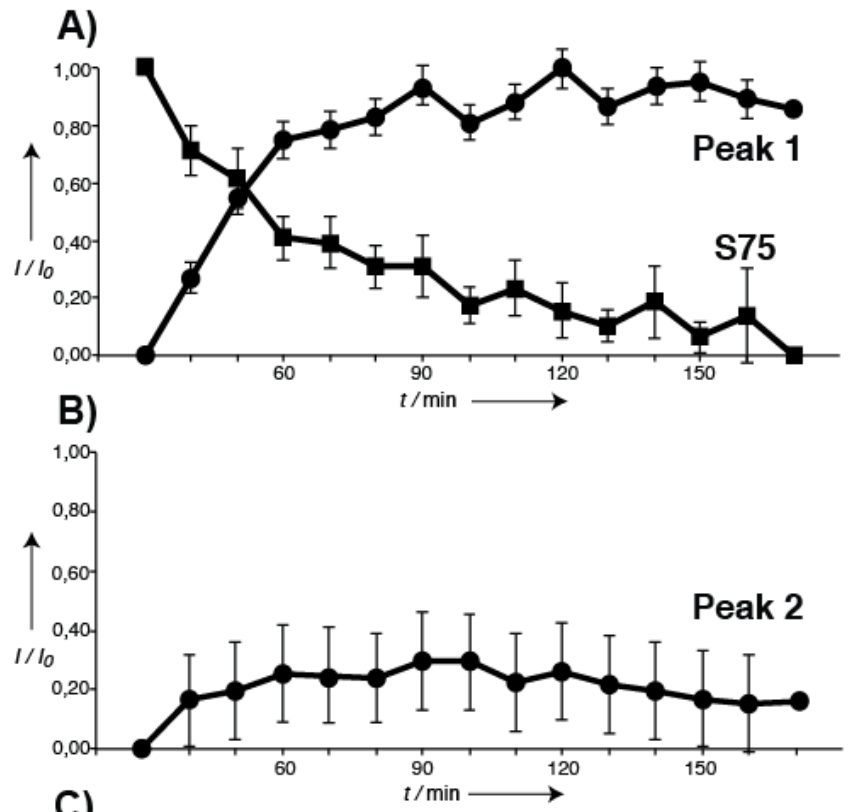

C)

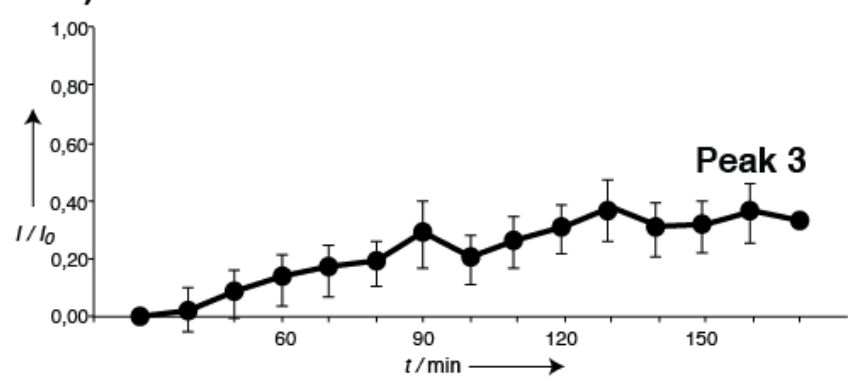

Figure 4. Time evolution of USrc phosphorylation in Xenopus egg extract (CSF) followed by NMR. A. S75 and pS75 (peak 1) normalized with respect to the maximum intensity of peak 1 ; $\mathbf{B}$. peak 2, tentatively assigned to pS69; C. pS17 (peak 3). Intensities were measured as running averages of two SOFASTHMQC experiments of 5 minutes each. The intensities of peaks 2 and 3 are shown scaled relative to the maximum intensity of peak $1\left(\mathrm{I}_{0}\right)$. Error bars show standard deviations propagated from the variations of peaks unaffected by phosphorylation between experiments. Protein concentration was $50 \mu \mathrm{M}$.

non-uniform sampling to achieve good resolution in the indirect dimension in a minimal time $\mathrm{e}^{[31],[32],[33]}$. Using our optimized conditions (see Experimental Section), we recorded well-resolved 2D spectra with acceptable signal to noise every 4 / 5 minutes from $50 \mu \mathrm{M}$ USrc samples in $X$. laevis egg extracts.

Peak 1 resonance appeared in the spectrum between 10 and 15 minutes after the addition of USrc to the extract. Peaks 2 and 3 were first observable in the spectra obtained between 15 and 20 minutes after the reaction started.

Figure $4 a$ shows the variation of peak intensities over time, for unmodified S75 signal and for peak 1 . Each intensity point is calculated as a running average of two experiments of 5 minutes each. After adding USrc to the extract, phosphorylation caused a decrease in the intensity of the S75 NH signal that matched the increase in peak 1 until a plateau was reached after 2 hours. 
The time evolution of the concentration of phosphorylated Ser/Thr residues of USrc in Xenopus egg extracts contributing to peak 2 and peak 3 intensities are shown in Figure $4 \mathrm{~b}$ and $4 \mathrm{c}$, respectively. The two peaks increased with different rates and more slowly than peak 1. In untreated extracts, the relative intensities of peaks 1,2 , and 3 were 1: 0.3: 0.5 respectively, after around 2 hours of reaction.

\section{USrc phosphorylation sites and active kinases in Xenopus egg extracts}

In order to assign the residues that are phosphorylated in the Xenopus egg extract we combined NMR, mass spectrometry and biochemical methods. The appearance of peak 1 paralleled the disappearance of the signal from unphosphorylated S75 suggesting that this peak corresponds to pS75. We had previously observed phosphorylation of USrc S75 by Cdk5 activated with p 25 by NMR in vitro $^{[28]}$. The phosphorylation of S75 was confirmed in USrC recovered after the NMR experiments with egg extracts by massspectrometry upon detection of pS75-specific tryptic and chymotryptic peptide MS/MS fragment ions, and by Western Blot using a specific anti phosphoS75-Src antibody (Fig. 5).

Treatment of the egg extracts with Roscovitine, an inhibitor of several CDKs including Cdk5, prevented the appearance of peaks 1 and 2 confirming that the corresponding phosphorylation events were catalyzed by CDKs (Fig. 6a). Cdk1 is known to be active in Xenopus egg extracts ${ }^{[30]}$. However, treatment of the extract with Ro3306, a selective inhibitor of Cdk1, had no effect on the phosphorylation of USrc detected by NMR (Fig. 6b). Western Blot analysis confirmed that phosphorylation of USrc on S75 was partially inhibited by adding Roscovitine but not Ro3306 to Xenopus egg extracts (Fig. 6g).

In vitro incubation of USrc with Cdk5/p25 resulted in the phosphorylation of both $\mathrm{S} 75$ and $\mathrm{T} 37^{[28]}$. However, phosphorylated T37 could not be detected by mass-spectrometry after incubation of USrc with Xenopus egg extracts. In contrast, mass-spectrometry unequivocally detected phosphorylated S69, although at lower abundance than pS75 (Fig.5 and Fig.S1, Supplementary material). Phosphorylation of residue $\mathrm{S} 69$ of $\mathrm{c}-\mathrm{Src}$ had previously been detected by MS in cell extracts from cancer lines HCT116 and MDAMB-435S ${ }^{[34]}$, although its function is presently unknown. S69 and S75 are present in the same tryptic peptide. Nevertheless, MS detected only mono-phosphorylated forms. The position of the phosphorylation site in these isobaric forms was deduced from exclusive peptide fragments in MS/MS experiments. Considering that the parent ion was mono-phosphorylated, fragments singlingout a potential site, phosphorylated or not, could be defined as exclusive fragments (Table 1, Supplementary material). A very rough estimate of the phosphorylation levels from the relative fragment intensities suggested a p69/p75 ratio of around 0.25 .


C)

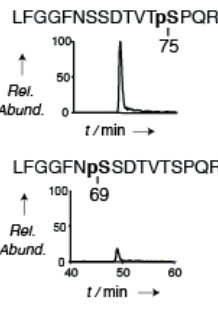

Figure 5. A. Extracted ion chromatograms of $\mathrm{m} / \mathrm{z} 463.2210$ (calculated $\mathrm{m} / \mathrm{z}$ 463.2208, $\Delta \mathrm{m}=0.43 \mathrm{ppm}$ ) (top) and 896.8997 (calculated $\mathrm{m} / \mathrm{z}$ 896.8987, $\Delta \mathrm{m}=1.11 \mathrm{ppm}$ ) (bottom) corresponding to RRpSLEPAENVH $(\mathrm{M}+3 \mathrm{H})^{3+}$ and $\mathrm{p}$-LFGGFNSSDTVTSPQR $(\mathrm{M}+2 \mathrm{H})^{2+}$ ions, respectively. B. Western blots showing phosphorylation of S17 and S75 of USrc in CSF extracts using specific antibodies. Protein loading was determined using an antibody against the Strep tag. C. The monophosphorylated peptide p-LFGGFNSSDTVTSPQR is a mixture of LFGGFNSSDTVTPSPQR and LFGGFNpSSDTVTSPQR that can be distinguished by MS/MS transitions $(896.89 \rightarrow 567.25$, y4) and $(896.89 \rightarrow 972.48$, y9-H2O) respectively.

The relative intensities of NMR peaks 2 and 1 were 0.32 suggesting that peak 2 may correspond to pS69. However, the appearance of this peak assigned to pS69 does not result in an observable decrease of the signal from the unmodified residue. This apparent contradiction could be accounted for by the observation of a general increase in the intensities caused by the phosphorylation of $\mathrm{S75}$, masking the small decrease expected by the (minor) phosphorylation of S69 (Fig.S2, Supplementary material). In fact, in the major species, the chemical shift of $S 69$ neighbour residues 66 , 67 and 70 were not affected (Fig. 3b) suggesting that this form is phosphorylated at $\mathrm{S} 75$ but not at $\mathrm{S} 69$. We interpreted the intensity increase of signals from the major species as a result of the elimination of residual interactions with the $60-75$ region caused by phosphorylation of S75, as previously observed ${ }^{[27]}$.

Peak 3 corresponds to phosphorylated serine 17 (pS17), which was phosphorylated by PKA in oocytes. The chemical shifts of phosphorylated residues are very sensitive to $\mathrm{pH}$ and the chemical shifts of phosphorylated residues observed in oocytes or oocyte extracts and in egg-extracts were not identical. However, the assignment was confirmed by the observation of a rapid increase in the intensity of peak 3 after the addition PKA to the egg extracts (Fig.S3, Supplementary material). After recovering USrc from the 
egg extract, the phosphorylation of residue S17 was confirmed by Western Blot, using a specific phospho-antibody. Phosphorylation at S17 was also confirmed by mass spectrometry (Fig. 5).

PKA is not the major kinase phosphorylating S17 of USrc in Xenopus egg extracts.

Although $\mathrm{S} 17$ can be phosphorylated by PKA, the addition of the PKA selective inhibitor PKA-I 6-22 (residues 6-22 of the PKA inhibitory protein $\mathrm{PKI}$ ) to CSF extracts did not impede the appearance of pS17 (Fig. 6c). Thus, an additional kinase present in the extract was able to catalyze S17 phosphorylation of USrc. However, the broader specificity inhibitor $\mathrm{H} 89$, which inhibits in addition to PKA other kinases such as S6K1, MSK1, ROCKII, PKBa and p90RSK prevented phosphorylation of $\mathrm{S} 17$, indicating that the kinase responsible for the phosphorylation of $\mathrm{S} 17$ in Xenopus egg extracts is sensitive to H89. Not surprisingly, Ro3306 had no effect on the phosphorylation of S17. Instead, addition of Roscovitine prevented the observation of peak 3 (pS17) by NMR, suggesting an indirect effect of inhibiting CDKs on the phosphorylation reaction on residue S17. Interestingly, the addition of PKA inhibitors PKA-I 6-22 and $\mathrm{H} 89$ also had a striking effect on the phosphorylation state of USrc residues targeted by CDKs: in the presence of PKA inhibitors the NMR signals corresponding to unmodified S75 was clearly observed and peaks 1 and 2 corresponding to phosphorylated species were not present (Fig. $6 \mathrm{c}$ and $6 \mathrm{~d}$ ). These results suggest that inhibition of PKA might trigger the activity of a phosphatase that reverses or strongly reduces the effect of CDKs keeping S75 unphosphorylated.

In order to test this hypothesis, H89 was added after a 30 minutes preincubation of USrc with the extract to allow the phosphorylation of USrc by CDKs. NMR measurements showed a decrease in the intensity of the CDK phosphorylated species following the addition of $\mathrm{H} 89$ (Figs. $6 \mathrm{e}$ and $6 \mathrm{f}$ ), indicating that the phosphatase activity compensates for the effect of the CDKs in the presence of the PKA inhibitor H89. The amount of phosphorylated S17 remained constant showing that the activated phosphatase is not affecting pS17. A strong decrease in the phosphorylation of S75 in Xenopus egg extracts caused by the addition of PKA-I 6-22 or H89 PKA was also observed using a specific pS75-Src antibody (Fig. 6g).

A candidate to mediate the effect of $\mathrm{H} 89$ is a member of the Protein Phosphatase 1 (PP1) inhibitor-1 (I-1) family. These ubiquitously distributed intrinsically disordered proteins are phosphorylated by PKA and become strong inhibitors of PP1. Inhibition of PKA would allow the dephosphorylation of I-1 and release of active PP1, leading to the observed absence of phosphorylation of S75.

Consistently, addition of PKA to the extract resulted, in addition to complete phosphorylation of S17, in an increase in the level of pS75. Interestingly, addition of PKA prevents the appearance of peak 2 (Fig.S3, Supplementary material). This effect could indicate that $\mathrm{S} 75$ and $\mathrm{S} 69$ are targets of a different set of kinases and phosphatases having different dependencies on PKA or may reflect that phosphorylation of $\mathrm{S} 69$ cannot occur after $\mathrm{S} 75$ is
D)

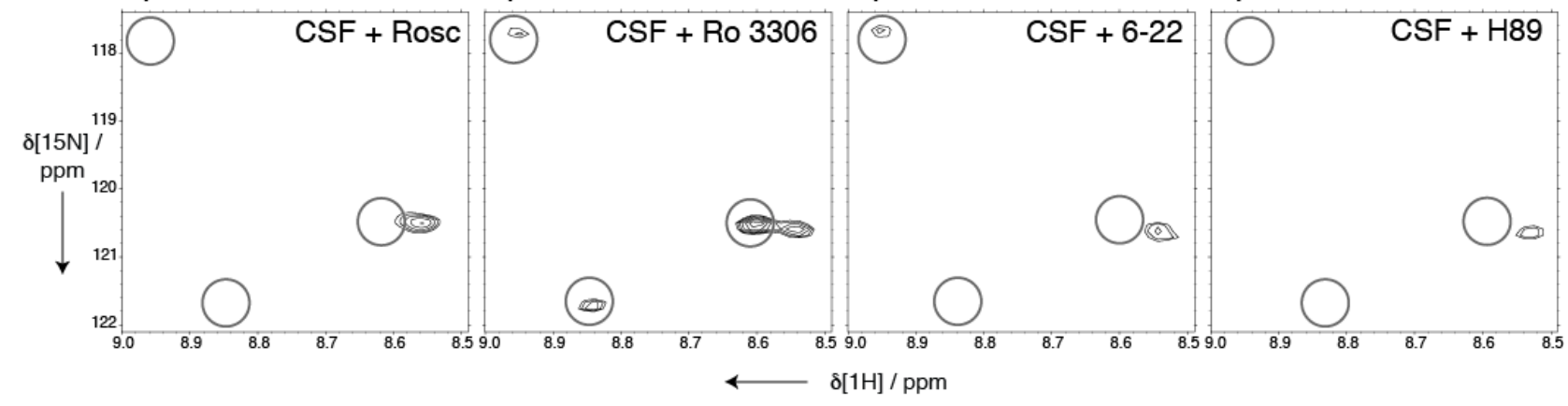

F)

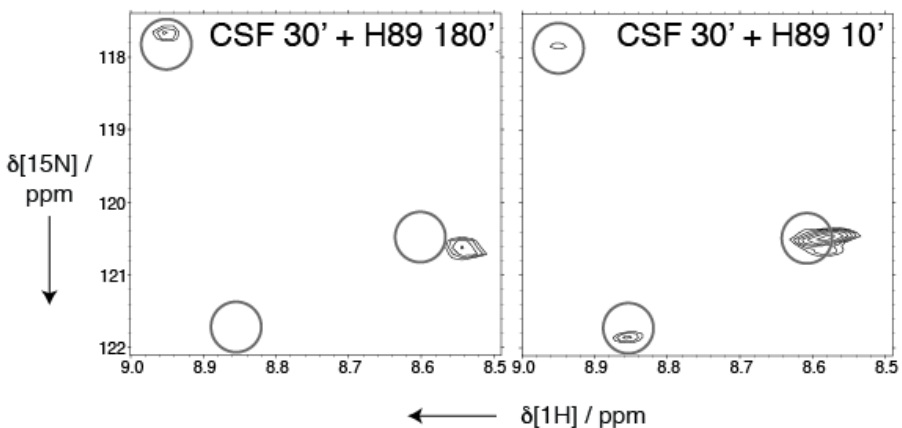

Figure 6. A-F. Expansions of ${ }^{1} \mathrm{H}-{ }^{15} \mathrm{~N}$ HSQC spectra of USrc in Xenopus egg extracts (CSF) treated with CDKs inhibitors Roscovitine (A) and Ro 3306 (B), and PKA inhibitors 6-22 fragment (C) and $\mathrm{H} 89$ (D). Panels $E$ and $\mathbf{F}$ compare expansions of ${ }^{1} \mathrm{H}-{ }^{15} \mathrm{~N}$ HSQC spectra of USrc at 10 and 180 minutes after the addition of $\mathrm{H} 89$ following preincubation in CSF for 30 minutes. G. Effects of CDKs/PKA inhibitors on S75 phosphorylation. USrc was isolated after being incubated with CSF extracts with added Roscovitine (0.5 mM); Ro 3306 (25 $\mu \mathrm{M}$ ); $6-22$ (50 $\mu \mathrm{M}$ ); H-89 (3 $\mu \mathrm{M})$. Membranes were immunoblotted with anti-Phospho Ser 75 (top panels) and anti-StrepTag (bottom panels). 
phosphorylated. Experiments using single point mutants under various conditions are currently in progress.

\section{Conclusions}

Real-time NMR spectroscopy allows the study of complex phosphorylation/dephosphorylation processes in the intrinsically disordered Unique domain of c-Src mediated by kinases and phosphatases that are present in Xenopus cells and cell extracts. The time course of the known phosphorylation in S17 and S75 could be studied and the not previously described (although detected) phosphorylation of S69 was unequivocally characterized by MS and assigned to one of the detected NMR signals. Addition of CDK or PKA kinase inhibitors or purified PKA clearly showed the cross-talk between kinases and phosphatases in Xenopus egg extracts, highlighting the use of in vivo NMR to study phosphatase, as well as kinase activities, and their mutual interplay. This approach is highly suited to characterize post-translational modifications in the intrinsically disordered regions that are present in most of the disease-related proteins in eukaryotic cells.

\section{Experimental Section}

\section{Cloning and Protein Expression and Purification}

The cDNA encoding (1-85) human c-Src region with a Strep-tag in C-terminal position for purification purposes was cloned into a $\mathrm{pET}$ 14b vector (Novagen, UK). The plasmid was transformed in Escherichia coli Rosetta ${ }^{\mathrm{TM}}$ (DE3) pLysS cells (Novagen, UK) and cells were grown in M9 minimal medium supplemented with $\left[{ }^{15} \mathrm{~N}\right] \mathrm{H}_{4} \mathrm{Cl}$ (Cambridge Isotope Laboratories, UK). USrc protein was isolated using Strep-tactin Sepharose (IBA, Göttingen). After elution with $2.5 \mathrm{mM}$ of desthiobiotin, it was further purified by size exclusion chromatography (Superdex 75 26/60, GE Healthcare, Spain) in phosphate buffer (50 mM phosphate, 0.2 mM EDTA, pH 7.0).

\section{Xenopus Oocytes}

$X$. laevis ovaries were surgically removed from full-grown females and treated with collagenase and dispase. Stage VI oocytes were selected and maintained in Barth's medium. Oocytes were microinjected with $50 \mathrm{nl}$ of purified ${ }^{15} \mathrm{~N}$-labeled USrc protein $(1 \mathrm{mM})$ and were left recovering for 3 hrs in modified Barth's medium at $18^{\circ} \mathrm{C}$ before NMR measurements.

For the preparation of lysates, oocytes were homogenized in $10 \mu \mathrm{l}$ per oocyte of ice-cold $\mathrm{H} 1 \mathrm{~K}$ buffer ( $80 \mathrm{mM} \beta$-glycerophosphate, $\mathrm{pH}$ 7.5, $20 \mathrm{mM}$ EGTA, $15 \mathrm{mM} \mathrm{MgCl}$, $1 \mathrm{mM}$ DTT, $1 \mathrm{mM}$ AEBSF, 2.5 $\mathrm{mM}$ Benzamidine, and $10 \mu \mathrm{g} / \mathrm{ml}$ each of Aprotinin and Leupeptin). Lysates were centrifuged at $10,000 \times \mathrm{g}$ for $10 \mathrm{~min}$ at $4{ }^{\circ} \mathrm{C}$, and the cleared supernatants were used for NMR measurements. Cytostatic factor (CSF) extracts were prepared with unfertilized $X$. laevis eggs. Protocols are detailed in ${ }^{[35], ~[36]}$. Immediately after preparation, CSF extracts were stored in aliquots of 100 or $200 \mu \mathrm{l}$ at $-80^{\circ} \mathrm{C}$.

\section{NMR sample preparation}

Cell-extract samples for NMR experiments were prepared by mixing $25 \mu \mathrm{l}$ of ${ }^{15} \mathrm{~N}$-labeled USrc protein (in $50 \mathrm{mM}$ phosphate, $0.2 \mathrm{mM}$ EDTA, pH 7.0, $0.5 \mathrm{mM}$ stock solution) and $25 \mu \mathrm{l}$ of $\mathrm{D}_{2} \mathrm{O}(10 \%)$ with $200 \mu \mathrm{l}$ of CSF or oocyte extracts. Prior to NMR measurements CSF extracts were thawed in ice and quickly span down. In order to minimize time for temperature equilibration in the spectrometer, $\mathrm{D}_{2} \mathrm{O}$ and ${ }^{15} \mathrm{~N}$-labeled protein stock solutions were kept between $16{ }^{\circ} \mathrm{C}$ and $18{ }^{\circ} \mathrm{C}$ before being added to the extract. Immediately after adding the protein to the extract, the samples ( $250 \mu$, final volume) were transferred to Shigemi tubes and inserted in the spectrometer for measurements.

The in-cell NMR samples were prepared as described above and details are in ${ }^{[5]}$. After recovering (see above), oocytes were first washed twice with MBS and, 30 minutes before NMR measurements, transferred to MBS containing $10 \% \mathrm{D}_{2} \mathrm{O}$. After microinjection, a dilution factor of around 20 in protein concentrations was expected, since the intracellular volume a one oocyte is of around $1 \mu$ l, resulting in a rise of concentration of the labelled protein of around $50 \mu \mathrm{M}$. Two hundred and fifty oocytes were used for individual in-cell NMR samples.

The following products were used for the different in vivo assays: Protein Kinase A (P2645-400UN, Sigma), H89 dihydrochloride (\#2910, Tocris Bioscience), Protein Kinase A Inhibitor Fragment 622 amide (P6062, Sigma), Ro 3306 (\#4181, Tocris Bioscience), and Roscovitine (\#557360, Calbiochem). Prior to the addition of the ${ }^{15} \mathrm{~N}$ labelled USrc, the kinases inhibitors were incubated 30 min with the CSF extract.

\section{Western Blotting}

USrc was recovered from CSF extracts by Strep-tag affinity purification after NMR measurements. $0.5 \mu \mathrm{g}$ of purified protein was separated by SDS-PAGE and transferred onto Hybond ECL nitrocellulose membrane (GE Healthcare). Membranes were blocked with $5 \%$ Milk in TBS-Tween and immunoblotted with the following antibodies: 1:1,000 anti-PhosphoSrc-Ser17 (\#5473, Cell Signaling Technology), 1:1,000 anti-PhosphoSrc-Ser75 (\#8A8186, AAT Bioquest) and 1:20,000 anti-Streptag antibody (IBA, Göttingen).

\section{Mass Spectrometry}

$20 \mu \mathrm{g}$ of ${ }^{15} \mathrm{~N}$-labeled USrc recovered from NMR experiments, or natural abundance USrc treated in identical way, was digested by adding trypsin or chymotrypsin $(2 \% \mathrm{w})$ and incubated at $37^{\circ} \mathrm{C}$ overnight. Digestions were stopped by adding formic acid to a final concentration of $1 \%$. The resulting peptide mixtures were diluted in $1 \% \mathrm{FA}$ and loaded in a nano-LC-MS/MS system connected to an Advion TriVersa NanoMate (Advion) fitted on an LTQ-FT Ultra mass spectrometer (Thermo Scientific). Further experimental details are given as supplementary information. 
A database search was performed with Bioworks v3.1.1 SP1 and Proteome Discoverer software v1.3 (Thermo Scientific) using Sequest search engine and SwissProt database, which included USrc protein and the common repository of adventitious proteins (http://www.thegpm.org/crap/index.html). Search parameters included no-enzyme specificity, methionine oxidation and phosphorylation in serine and threonine as dynamic modification and, depending on the sample, amino acids labeled with ${ }^{15} \mathrm{~N}$ as static modification.

Extracted ion chromatograms of MS or MS/MS ions were obtained using Xcalibur software vs 2.0SR2 (Thermo Scientific).

\section{NMR Spectroscopy}

NMR experiments of ${ }^{15} \mathrm{~N}$ labeled USrc in $50 \mathrm{mM}$ phosphate buffer $\mathrm{pH} 7.0$ were recorded at $288 \mathrm{~K}$ with a $600 \mathrm{MHz}$ spectrometer equipped with a $\mathrm{TCl}$ cryo-probe at $0.5 \mathrm{mM}$ protein concentration. NMR experiments in live oocytes as well as in the cellular extracts were performed at $288 \mathrm{~K}$, which is the optimal temperature for live oocytes, and $50 \mu \mathrm{M}$ concentration.

USrc resonance assignment had been previously reported. Combined $\mathrm{NH}$ chemical shift differences were computed as in equation (1):

$$
\Delta \delta=\left[\Delta \delta_{H}^{2}+\left(\Delta \delta_{N} / 5\right)^{2}\right]^{1 / 2}
$$

where $\delta_{H}$ and $\delta_{N}$ are the changes in chemical shift for ${ }^{1} \mathrm{H}$ and ${ }^{15} \mathrm{~N}$, respectively. In vitro kinase reactions were reconstituted with $50 \mu \mathrm{M}$ of ${ }^{15} \mathrm{~N}$ - protein and recombinant PKA, 400 enzymatic units, in the presence of ATP $(0.5 \mathrm{mM})$ and $\mathrm{Mg}_{2} \mathrm{SO}_{4}(0.1 \mathrm{mM})$.

In buffer and in cell NMR experiments were performed in Shigemi tubes. For experiments with cell extracts Shigemi or $3 \mathrm{~mm}$ NMR tubes were used. Probe tuning and shimming was performed in a dummy sample equilibrated at $288 \mathrm{~K}$ immediately before the insertion of the real sample to minimize dead time in the real time measurements setting. The time from protein addition to the start of NMR data acquisition was $5 \mathrm{~min}$. For quantitative time-resolved experiments, we acquired consecutive ${ }^{1} \mathrm{H}-{ }^{15} \mathrm{~N}$ SOFAST-HMQC $\mathrm{C}^{[31]}$ correlation spectra while the kinase reaction proceeded. The data were recorded with 4 or 8 transients and $800\left({ }^{1} \mathrm{H}\right)-256$ or $512\left({ }^{15} \mathrm{~N}\right)$ complex points, in traditional or non-uniform sampling scheme. Spectral width in the ${ }^{15} \mathrm{~N}$ dimension was minimized in order to improve resolution in the indirect dimension. NMR data were processed with NMRPipe ${ }^{[37]}$ or qMDD $^{[33]}$ software and spectra were analyzed with Sparky ${ }^{[38]}$.

\section{Competing Financial Interests}

The authors declare no competing financial interests.

\section{Acknowledgements}

We thank Philip Selenko and Volker Dötsch for help in the initial implementation of the Xenopus oocyte experiments. This work was supported by funds from the Spanish MICINN-FEDER (BIO201015683 and BFU2010-17850), Fundació Marató TV3, the Generalitat de Catalunya (2009SGR1352) and the European Union 7FP (Bio-NMR contract 261863 and Marie Curie Action (COFUND) to I.A.) A.R.N. acknowledges support by the Fundación BBVA. NMR instruments were made available by the Spanish NMR "Instalación Científico Tecnológica Singular" of the University of Barcelona.

Keywords: in cell NMR $\cdot$ MS $\cdot$ phosphoproteins $\cdot \mathrm{c}$-SrC $\cdot$ Intrinsically Disordered Proteins

[1] P. Selenko, D. P. Frueh, S. J. Elsaesser, W. Haas, S. P. Gygi, G. Wagner, Nature Structural \& Molecular Biology 2008, 15 , 321-329.

[2] F.-X. Theillet, C. Smet-Nocca, S. Liokatis, R. Thongwichian, J. Kosten, M.-K. Yoon, R. W. Kriwacki, I. Landrieu, G. Lippens, P. Selenko, Journal of Biomolecular NMR 2012, 217-236.

[3] J.-T. Du, Y.-M. Li, W. Wei, G.-S. Wu, Y.-F. Zhao, K. Kanazawa, T. Nemoto, H. Nakanishi, Journal of the American Chemical Society 2005, 127, 16350-16351.

[4] P. Selenko, G. Wagner, Nature Methods 2006, 3, 80-81.

[5] P. Selenko, Z. Serber, B. Gadea, J. Ruderman, G. Wagner, Proceedings of the National Academy of Sciences of the United States of America 2006, 103, 11904-11909.

[6] P. Selenko, G. Wagner, Journal of Structural Biology 2007, 158, 244-253.

[7] P. Bernadó, J. García de la Torre, M. Pons, Journal of Molecular Recognition: JMR 2004, 17, 397-407.

[8] G. J. Pielak, C. Li, A. C. Miklos, A. P. Schlesinger, K. M. Slade, G.-F. Wang, I. G. Zigoneanu, Biochemistry 2009, 48, 226-234.

[9] J. J. Ward, J. S. Sodhi, L. J. McGuffin, B. F. Buxton, D. T. Jones, Journal of Molecular Biology 2004, 337, 635-645.

[10] C. J. Oldfield, Y. Cheng, M. S. Cortese, C. J. Brown, V. N. Uversky, a K. Dunker, Biochemistry 2005, 44, 1989-2000.

[11] Y. Naranjo, M. Pons, R. Konrat, Molecular bioSystems 2012, 8, 411-416.

[12] A K. Dunker, I. Silman, V. N. Uversky, J. L. Sussman, Current Opinion in Structural Biology 2008, 18, 756-764.

[13] V. N. Uversky, C. J. Oldfield, U. Midic, H. Xie, B. Xue, S. Vucetic, L. M. lakoucheva, Z. Obradovic, a K. Dunker, BMC genomics 2009, 10 Suppl 1, S7.

[14] A. Stein, R. a Pache, P. Bernadó, M. Pons, P. Aloy, The FEBS journal 2009, 276, 5390-5405.

[15] M. T. Brown, J. A. Cooper, Biochimica et Biophysica Acta 1996, 1287, 121-149.

[16] S. M. Thomas, J. S. Brugge, Annual Review of Cell and Developmental Biology 1997, 13, 513-609.

[17] G. S. Martin, Nature Reviews. Molecular Cell Biology 2001, 2, 467-475. 
[18] S. J. Parsons, J. T. Parsons, Oncogene 2004, 23, 7906-7909.

[19] T. J. Yeatman, Nat. Rev. Cancer 2004, 4, 470-480.

[20] K. Hansen, G. Alonso, S. a Courtneidge, L. Rönnstrand, C. H. Heldin, Biochemical and Biophysical Research Communications 1997, 241, 355-362.

[21] T. M. Johnson, N. a Williamson, G. Scholz, a Jaworowski, R. E. Wettenhall, a R. Dunn, H. C. Cheng, The Journal of Biological Chemistry 2000, 275, 33353-33364.

[22] I. Joung, T. Kim, L. a Stolz, G. Payne, D. G. Winkler, C. T. Walsh, J. L. Strominger, J. Shin, Proceedings of the National Academy of Sciences of the United States of America 1995, 92, 5778-5782.

[23] Y. Obara, K. Labudda, T. J. Dillon, P. J. S. Stork, Journal of Cell Science 2004, 117, 6085-6094.

[24] G. Kato, S. Maeda, Journal of Biochemistry 1999, 126, 957961.

[25] G. Kato, Journal of Biochemistry 2003, 133, 563-569.

[26] Q. Pan, F. Qiao, C. Gao, B. Norman, L. Optican, P. S. Zelenka, Cellular and Molecular Life Sciences: CMLS 2011, 68, 3425-3436.

[27] Y. Pérez, M. Maffei, A. Igea, I. Amata, M. Gairí, A. R. Nebreda, P. Bernadó, M. Pons, Scientific Reports 2013, 3, 1295, DOI:10.1038/srep01295.

[28] Y. Pérez, M. Gairí, M. Pons, P. Bernadó, Journal of Molecular Biology 2009, 391, 136-148.

[29] A. Schmitt, A. R. Nebreda, Journal of Cell Science 2002, 115, 2457-2459.

[30] J. E. Ferrel, BioEssays: news and reviews in molecular, cellular and developmental biology, 1999, 21, 833-842.

[31] P. Schanda, E. Kupce, B. Brutscher, Journal of Biomolecular NMR 2005, 33, 199-211.
[32] K. Kazimierczuk, V. Y. Orekhov, Angewandte Chemie (International Ed. in English) 2011, 50, 5556-5559.

[33] V.Y. Orekhov, V.A. Jaravine, Prog. Nucl. Magn. Reson. Spectrosc., 2011, 59, 271-292.

[34] F. S. Oppermann, F. Gnad, J. V Olsen, R. Hornberger, Z. Greff, G. Kéri, M. Mann, H. Daub, Molecular \& Cellular Proteomics : MCP 2009, 8, 1751-1764.

[35] E. Perdiguero, A.R. Nebreda, Methods Mol Biol. 2004, 250, 299-314.

[36] Z. Serber, P. Selenko, R. Haensel, S. Reckel, F. Loehr, J. E. Ferrel Jr, G. Wagner \& V. Doetsh, Nature Protocols. 2006, 1, 2701-2709.

[37] F. Delaglio, S. Grzesiek, G. W. Vuister, G. Zhu, J.Pfeifer and A. Bax, J. Biomol. NMR. 1995, 6, 277-293.

[38] T.D. Goddard and D.G. Kneller, SPARKY 3, University of California, San Francisco.

Received: ((will be filled in by the editorial staff))

Published online: ((will be filled in by the editorial staff)) 
Entry for the Table of Contents

Layout 1:

\section{FULL PAPERS}

Real time in cell NMR reveals complex phosphorylation dynamics of the Unique domain of $\mathrm{c}$-Src

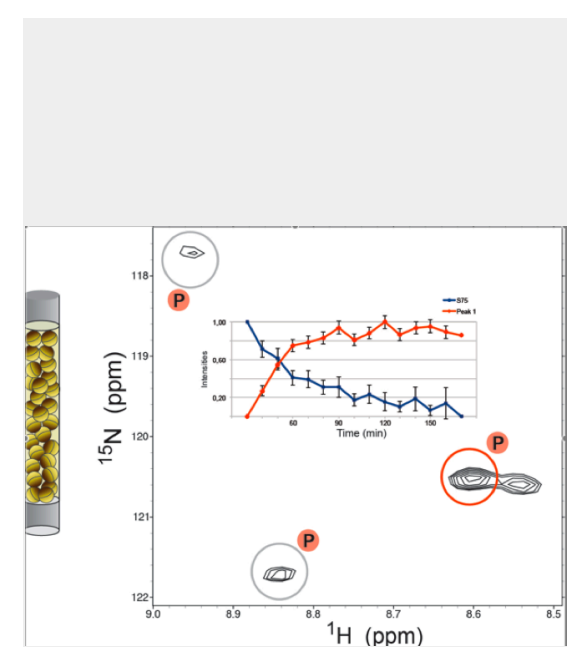

Irene Amata, Mariano Maffei, Ana Igea,

Marina Gay, Marta Vilaseca, Angel R. Nebreda*, Miquel Pons*

Page No. - Page No.

Multi-phosphorylation Of The Intrinsically Disordered Unique

Domain Of c-Src Studied By In-cell and Real-Time NMR 